\title{
Motion Planning by Demonstration with Human-Likeness Evaluation for Dual-Arm Robots
}

\author{
Néstor García, Jan Rosell and Raúl Suárez
}

\begin{abstract}
The paper presents a planning procedure that allows an anthropomorphic dual-arm robotic system to perform a manipulation task in a natural human-like way by using demonstrated human movements. The key idea of the proposal is to convert the demonstrated trajectories into attractive potential fields defined over the configuration space and then use an RRT*-based planning algorithm that minimizes a path-cost function designed to bias the tree growth towards the human-demonstrated configurations. The paper presents a description of the proposed approach as well as results from a conceptual and a real application example, the latter using a real anthropomorphic dual-arm robotic system. A path-quality measure, based on first-order synergies (correlations between joint velocities) obtained from real human movements, is also proposed and used for evaluation and comparison purposes. The obtained results show that the paths obtained with the proposed procedure are more human-like.
\end{abstract}

Index Terms-Path planning for manipulators, Humanoid robots, Synergies, Human-Like motions.

\section{INTRODUCTION}

$\mathbf{M}$ OTION planning is a basic research issue in robotics, particularly since the robots became an essential part in many application fields like, for instance, the medical and the electronic industries, or even in the computational biology or computer animation fields. The importance of this problem is more relevant for robotic systems with a high number of degrees of freedom (DOFs), like those involving mechanical hands or anthropomorphic structures. Moreover, a path is sometimes required that, besides being collision-free, also optimizes a quality measure like minimizing the traveled distance [1] or the time required in the path execution [2]. In the case of humanoid robots, one of the needs is to find robot movements that mimic those of human beings, since human-robot collaboration is facilitated if the robot shows human-like movements [3] because when humans are familiar with the robot motions, they may adjust their motions to avoid possible collisions or enhance the collaboration.

Different planning algorithms able to tackle the motion planning of complex systems have been developed, being the sampling-based planners the most outstanding [4]. Among them, the most commonly used are the Probabilistic Roadmaps (PRM) [5] and the Rapidly-exploring Random Trees (RRT) [6]. Diverse improvements have been proposed to these planners to deal with constraints [7], to consider

The authors are with the Institute of Industrial and Control Engineering (IOC), Universitat Politècnica de Catalunya (UPC), Barcelona, Spain, (\{nestor.garcia.hidalgo, jan.rosell, raul.suarez\}@upc.edu). This work was partially supported by the Spanish Government through the projects DPI2013-40882-P, DPI2014-57757-R and DPI2016-80077-R. N. García is supported by the Generalitat de Catalunya through the grant FI-DGR 2016. configuration-space costmaps [8], or to bias the sampling towards better regions of the configuration space by using, for instance, retraction-based methods [9] or Principal Component Analysis (PCA) [10]. Since these algorithms are non-optimal, other variants like the $\mathrm{PRM}^{*}$ and RRT* algorithms have been proposed [11]. Besides, recently, the RRT* planner was combined with potential fields in order to improve its efficiency [12] and guide the solution path [13]. In this line, this paper proposes the use of a variant of the RRT* algorithm that minimizes a path-cost function computed with a potential field obtained from human demonstrations, thus resulting in human-like motions. Potential functions learned from human demonstrations have been also used in control policies [14].

Looking for human-like movements leads to the search of the right coordination between the robot joint movements. Towards this goal, the direct use of the real movements of a human being as a reference is common [15]. On the one hand, a usual assumption of many approaches analyzing human motion is that humans try to minimize an unknown cost function while doing everyday manipulation tasks, e.g. hand jerk (i.e. the third time-derivative of the hand position) [16], joint jerk [17], joint torque [18] or a convex combination of several cost functions with weighting factors chosen to describe an observed human motion [19]. Hence, minimizing these cost functions, human-like motions can be obtained. However, it can be complex to incorporate these functions in the inverse kinematics of some manipulators. On the other hand, new human-like movements can be obtained using human motions previously registered, e.g. by properly modifying these motions to fit a new scenario [20], by using these known movement trajectories to train a neural network [21] or to adjust the parameters of a non-linear dynamical system [22]. Other related advanced procedures include e.g. the generation of cyclic motions for dual-arm robots using neural networks and quadratic programing [23], the consideration of the robot dynamics in the motion planning by transforming the problem into an optimization of a non-linear fitness function [24] or the use of movement primitives based on a model of the triangle defined by the human upperarm and forearm [25].

In the motion planning of mechanical hands, several works used "postural synergies" (i.e. correlations between DOFs) to simplify the problem by reducing the dimension of the search space as well as to mimic human postures. The correlations of the human hand configurations while performing a grasp were studied [26] and mapped into a robotic hand [27]. These synergies were suggested to be an emergent consequence of neuromuscular impedance [28]. The synergies existing in the human hand [29] were also used for other objectives such as the analysis and design of robotic hands in order to mimic 


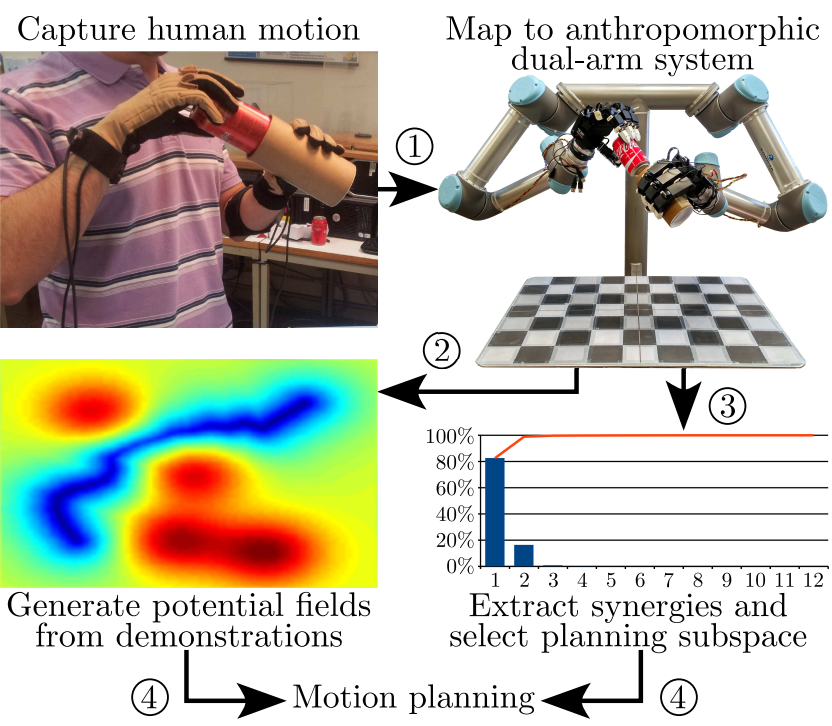

Fig. 1. General schema of the proposed approach.

human grasps [30], the selection of grasping forces [31] and the design of specific hand control systems [32]. Later, a compliant model for synergies, called "soft synergies", was introduced and used in the selection of grasping forces, in their control, and in the control of the motion of the grasped object [33], [34]. The use of synergies was recently also used in a dual-arm anthropomorphic system while performing manipulation tasks [35], [36]. One of the main problems faced when trying to obtain human synergies is the capture of the human configurations in order to get proper information for the search of the synergies. In the case of the hands mentioned above, the problem was mainly addressed using: a) vision systems (e.g. [37]), which have the frequent problem of visual occlusions and usually require special marks on the hand to facilitate the configuration identification, and b) sensorized gloves (e.g. [38]). In the particular application of moving a prosthetic hand, the analysis of forearm electromyogram signals (EMG) was proposed (e.g. [39]) but this is not practical in most general-purpose robotic applications.

The works mentioned above dealt with synergies involving correlations between joint positions. Nevertheless, a recent work extends the concept of synergies to the velocity space (i.e. the space of the first derivative of the configuration trajectories) calling them first-order synergies [40], [41] (in contrast with the synergies in the configuration space, that were called zero-order synergies). In this line, this paper proposes a human-likeness index based on first-order synergies and uses it to evaluate the paths obtained with the proposed motion planner based on human demonstrations.

After this introduction, Section $\amalg$ presents the problem statement and the approach overview, and Section III outlines the required preliminaries. Then, Section IV] details the proposal of the planner and its performance analysis, and Section $\mathrm{V}$ introduces the human-likeness index and uses it to evaluate the proposed planner. Finally, Section VI presents the conclusions and future work.

\section{Motivation AND APPROACH OVERVIEW}

The first goal of this work is to introduce a planning procedure designed specifically for anthropomorphic dual-arm robotic systems that solves manipulation tasks using human-like paths. To this end, the movements of a human operator are used as demonstration paths. The main features of the proposed approach are the following (see Fig. 11):

1) The movements of a human operator solving manipulation tasks are captured and then mapped to the anthropomorphic dual-arm robotic system.

2) The demonstration paths are used to generate attractive potential fields over the configuration space $\mathcal{C}$.

3) The captured movements are also used to select a region of a lower-dimensional subspace of $\mathcal{C}$, called $B_{k}$, that contains a predefined high percentage of the sample variance of the demonstration paths. By planning in this subspace, a significant reduction of the computational cost is expected.

4) Using the potential fields generated in $\mathcal{C}$, a path cost is defined to guide an RRT ${ }^{*}$-based planner. The proposed planning algorithm uses a stochastic gradient-descent method to minimize the path cost and to bias the tree growth towards the demonstrated human movements.

The second goal of the work is to define a quality index to evaluate the human-likeness of a path by considering how much aligned the path is with respect to certain reference human movements. These movements, which can be different to the ones used as demonstration paths to solve the task, are characterized by using a set of first-order synergies defined over the relevant configurations of $\mathcal{C}$.

Note that, since the two goals are clearly independent, we have addressed them separately in Section IV and Section $\mathrm{V}$ respectively, each one with its own experimentation. Thereby, first a path is obtained with the proposed planning algorithm using demonstration paths (Section IV), and then the human-likeness of the planned path is evaluated (Section $\mathrm{V}$; this human-likeness index can be used to evaluate the paths obtained with the proposed planning procedure or with any other motion planner.

\section{PRELIMINARIES}

This section presents some basic concepts and procedures (introduced in previous works of the authors [13], [35], [40]) that are relevant to the present proposal.

\section{A. Capturing and mapping human motions}

Human movements are used to guide the motion planning to find human-like paths for dual-arm systems and to evaluate the human-likeness of the paths. Human movements can be captured and mapped to the robot configuration space in different ways, depending on the available sensors and on the robot kinematics. In this work, human movements are captured using magnetic trackers and sensorized gloves, as shown in the top-left picture in Fig. 1, that take samples of the position and orientation of the operator wrists while performing manipulation tasks. Then, the captured data are mapped to the dual-arm 
robotic system by solving the inverse kinematics of the arms for each sampled wrist configuration, obtaining in this way the corresponding configurations of the dual-arm system. Inverse kinematics of robotic arms usually have several solutions, or even infinite in the case of redundant arms with more than six DOFs, therefore some anthropomorphism criterion should be used to solve it (e.g. controlling the position of the robot elbows [42]).

In this way, for each task execution done by the operator, a sequence of configuration samples is obtained in the robot configuration space $\mathcal{C}$, defining a sequence $\mathcal{P}_{i}$ of rectilinear segments connecting time-consecutive mapped configurations. In our case, the dual-arm system used is composed of two UR5 6-DOF robotic arms from Universal Robots, assembled emulating the human arm configuration as shown in the top-right picture in Fig. 1, each arm being equipped with a 16-DOF Allegro Hand from Simlab (although the present paper is only focused in the motions of the arms).

\section{B. Zero- and first-order synergies}

The postural synergies are correlations between the joint positions of an articulated system [30]. This widely used concept was called zero-order synergies in [40], where the extension to the joint velocity space was proposed and the correlations between velocities was called first-order synergies. The zero-order synergies are obtained from the Principal Component Analysis (PCA) of a set of captured configuration samples. This returns a new basis of $\mathcal{C}$, called zero-order basis ${ }^{0} S$, with the axes ordered according to the sample dispersions along them. Each axis represents a zero-order synergy and the movement along it, equivalent to a single DOF, implies the correlated movement of several (or all) the actual DOFs of the system. The procedure to obtain the first-order synergies is exactly the same as the one to obtain zero-order synergies, but in this case using velocity samples. Thereby, the new bases of the velocity space, defined by a barycenter $\boldsymbol{\mu}$ and a covariance matrix $\Sigma$ of velocity samples, are called first-order bases, ${ }^{1} S$. Each axis of a basis ${ }^{1} S$ is called a first-order synergy.

The linear approximation of the PCA is enough to represent the subspace where the demonstrated motions lie. In fact, it has been demonstrated to be useful and implementable by a drive mechanism [43] or a real-time algorithm [44]. However, nonlinear approaches to obtain synergies have been also proposed, such as the Gaussian process latent variable model [26] and the unsupervised kernel regression [45].

The zero-order synergies are used to detect the relevant region of $\mathcal{C}$, called box $B\left({ }^{0} S_{G}\right)$, where the captured motions take place. Notice that the directions of the human motions depend on the region of the configuration space where it takes place. Therefore, to take this into account, the box $B\left({ }^{0} S_{G}\right)$ is divided into subregions where the ${ }^{1} S$ bases are significantly different, as introduced in [40]. As an example, Fig. 2 shows the box $B\left({ }^{0} S_{G}\right)$ and its resulting partition based on differences of first-order synergies for the samples of a motion that follows a ray shape and for the samples of another motion that follows an elliptic trajectory in clock-wise sense. For the ray-shaped motion, $B\left({ }^{0} S_{G}\right)$ is split into two parts, where the motion

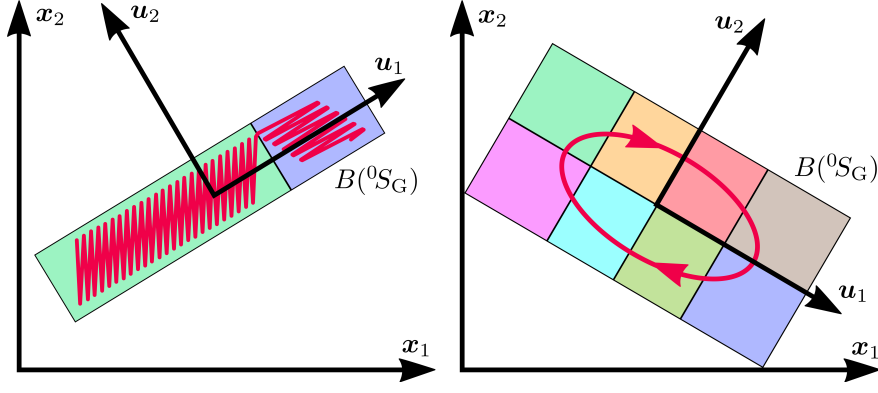

Fig. 2. A ray-shaped motion (left) and an elliptic motion (right), shown in red, with the resulting cell-decomposition of $\mathcal{C}$ based on the first-order synergy differences. Boxes $B\left({ }^{0} S_{G}\right)$ have been split into two and eight cells, respectively, with planes aligned with the ${ }^{0} S_{G}$ axes (i.e. $\boldsymbol{u}_{1}$ and $\boldsymbol{u}_{2}$ ).

directions differ significantly from each other; and for the elliptic motion, $B\left({ }^{0} S_{G}\right)$ is split into eight cells.

Note that first-order synergies always exist if the sampled joint values are not homogeneously distributed, which is quite unlike in real human movements (that is why the first-order synergies are useful in the analysis of human movements).

In the present work:

- The ${ }^{0} S$ basis is used to define the lower-dimensional subspace where the planning will be done (Section [V].

- The ${ }^{1} S$ bases are used to define a human-likeness index to evaluate the solution paths found for the dual-arm robotic systems (Section V).

\section{Motion-cost function}

An RRT*-based planner recently proposed allows the user to guide the tree growth in a simple and transparent way [13]; this is done by defining attractive and repulsive points and segments in the workspace that generate a potential field $V(\boldsymbol{q})$ in the configuration space $\mathcal{C}$. Then, the planner constructs low-cost paths following the resulting valleys and saddle points in $\mathcal{C}$. Considering piece-wise linear paths in $\mathcal{C}$, the path cost is computed by adding the costs of the rectilinear segments (called motions). The cost of a motion between two configurations $\boldsymbol{q}_{\mathrm{i}}$ and $\boldsymbol{q}_{\mathrm{f}}$ is defined as the linear combination of three other costs $c_{\mathrm{P}}, c_{\mathrm{I}}$ and $c_{\mathrm{D}}$ with respective positive weights $\omega_{\mathrm{P}}, \omega_{\mathrm{I}}$ and $\omega_{\mathrm{D}}$ :

$$
c\left(\boldsymbol{q}_{\mathrm{i}}, \boldsymbol{q}_{\mathrm{f}}\right)=\omega_{\mathrm{P}} \underbrace{\left\|\boldsymbol{q}_{\mathrm{f}}-\boldsymbol{q}_{\mathrm{i}}\right\|}_{c_{\mathrm{P}}\left(\boldsymbol{q}_{\mathrm{i}}, \boldsymbol{q}_{\mathrm{f}}\right)}+\underbrace{\omega_{\boldsymbol{q}_{\mathrm{i}}}^{\int_{V(\boldsymbol{q})}^{\boldsymbol{q}_{\mathrm{f}}} \mathrm{d} \boldsymbol{q}}}_{c_{\mathrm{I}}\left(\boldsymbol{q}_{\mathrm{i}}, \boldsymbol{q}_{\mathrm{f}}\right)}+\underbrace{\int_{\boldsymbol{q}_{\mathrm{i}}}^{\boldsymbol{q}_{\mathrm{f}}\left|\frac{\partial V(\boldsymbol{q})}{\partial \boldsymbol{q}}\right| \mathrm{d} \boldsymbol{q}}}_{c_{\mathrm{D}}\left(\boldsymbol{q}_{\mathrm{i}}, \boldsymbol{q}_{\mathrm{f}}\right)}
$$

where $c_{\mathrm{P}}$ calculates the motion length, $c_{\mathrm{I}}$ measures the motion effort, computed as the product of the average value of $V(\boldsymbol{q})$ and the motion length, and $c_{\mathrm{D}}$ evaluates the variations of $V(\boldsymbol{q})$ along the motion. Therefore, the path minimizing this motion-cost function connects the start and the goal configurations in the shortest way that avoids the areas with high $V(\boldsymbol{q})$ values (i.e. with repulsive potential fields) and, at the same time, keeps $V(\boldsymbol{q})$ as monotonic as possible along the path (i.e. avoiding unneeded motions from repulsive to attractive potential fields and vice versa). 
In the present work, the potential field is generated using real human movements and the motion-cost function guides the solution path towards these demonstrated human motions.

\section{PROPOSED MOTION PLANNING PROCEDURE}

\section{A. Generating potential fields from demonstrations}

The proposed planning algorithm tries to follow the demonstrated human movements by minimizing the cost of the path over the configuration space $\mathcal{C}$, where several potential fields are defined to guide the tree growth. For this, the demonstration paths generate attractive potential fields. In addition, the goal configuration $\boldsymbol{q}_{\text {goal }}$ acts also as an attractor. On the other hand the obstacles generate potential fields that repulse the robotic arms while the arms also repel each other. To compute the potential-field value $V(\boldsymbol{q})$, let first:

$\lambda \geq 0$ and $\sigma \geq 0$ be, respectively, the strength and the diffusion parameters of each potential field;

$\mathcal{P}_{i} \in P$ be the $i$-th path of the set of demonstration paths $P$, obtained from the mapping of the human movements and projected onto $\mathcal{C}$;

$\mathcal{O}_{j} \in O$ be the $j$-th obstacle of the set of obstacles $O$ with which the dual-arm system can collide;

$\mathrm{d}\left(\boldsymbol{q}, \mathcal{P}_{i}\right)$ be the minimum distance in $\mathcal{C}$, between the configuration $\boldsymbol{q}$ and the demonstration path $\mathcal{P}_{i}$;

$\mathrm{d}\left(\mathcal{L}_{\boldsymbol{q}}, \mathcal{R}_{\boldsymbol{q}}\right)$ be the minimum distance in the workspace between both arms of the robotic system, when the dual-arm robot configuration is $\boldsymbol{q}$;

$\mathrm{d}\left(\mathcal{L}_{\boldsymbol{q}}, \mathcal{O}_{j}\right)$ and $\mathrm{d}\left(\mathcal{R}_{\boldsymbol{q}}, \mathcal{O}_{j}\right)$ be the minimum distances in the workspace between the obstacle $\mathcal{O}_{j}$ and the left arm and the right arm, respectively, when the dual-arm robot configuration is $\boldsymbol{q}$.

Then, the resultant potential-field value $V(\boldsymbol{q})$ at a collision-free configuration $\boldsymbol{q}$ is defined as the sum of four potential fields values:

$$
V(\boldsymbol{q})=V_{\text {goal }}(\boldsymbol{q})+V_{\text {paths }}(\boldsymbol{q})+V_{\text {arms }}(\boldsymbol{q})+V_{\text {obs }}(\boldsymbol{q})
$$

where:

$$
V_{\text {goal }}(\boldsymbol{q})=\lambda_{\text {goal }}\left(1-e^{-\sigma_{\text {goal }}\left\|\boldsymbol{q}-\boldsymbol{q}_{\text {goal }}\right\|^{2}}\right)
$$

is the potential-field value of the configuration $\boldsymbol{q}$ regarding the attractive potential field of the goal configuration $\boldsymbol{q}_{\text {goal }}$ (i.e. the closer are $\boldsymbol{q}$ and $\boldsymbol{q}_{\text {goal }}$, the smaller is $\left.V_{\text {goal }}\right)$;

$$
V_{\text {paths }}(\boldsymbol{q})=\sum_{i}^{|P|} \frac{\lambda_{i}}{|P|}\left(1-e^{-\sigma_{i} \mathrm{~d}\left(\boldsymbol{q}, \mathcal{P}_{i}\right)^{2}}\right)
$$

is the potential-field value of the configuration $\boldsymbol{q}$ regarding the attractive potential fields generated by all the demonstration paths $\mathcal{P}_{i} \in P$, i.e. $V_{\text {paths }}$ decreases when $\boldsymbol{q}$ gets closer to $P$ (it must be noted that, since $\mathcal{P}_{i}$ and $\boldsymbol{q}$ are both expressed in $\mathcal{C}, \mathrm{d}\left(\boldsymbol{q}, \mathcal{P}_{i}\right)$ is simply the minimum Euclidean distance in $\mathcal{C}$ between $\boldsymbol{q}$ and the rectilinear segments representing $\mathcal{P}_{i}$ );

$$
V_{\text {arms }}(\boldsymbol{q})=\lambda_{\text {arms }} e^{-\sigma_{\text {arms }} \mathrm{d}\left(\mathcal{L}_{\boldsymbol{q}}, \mathcal{R}_{\boldsymbol{q}}\right)^{2}}
$$

is the potential-field value of the configuration $\boldsymbol{q}$ regarding the repulsive potential field between the arms of the robotic system, i.e. $V_{\text {arms }}$ grows if the arms get closer; and

$$
V_{\text {obs }}(\boldsymbol{q})=\sum_{j=1}^{|O|} \frac{\lambda_{j}}{|O|}\left(e^{-\sigma_{j} \mathrm{~d}\left(\mathcal{L}_{\boldsymbol{q}}, \mathcal{O}_{j}\right)^{2}}+e^{-\sigma_{j} \mathrm{~d}\left(\mathcal{R}_{\boldsymbol{q}}, \mathcal{O}_{j}\right)^{2}}\right)
$$

is the potential-field value of the configuration $\boldsymbol{q}$ regarding the repulsive potential fields of all the obstacles $\mathcal{O}_{j} \in O$, if either the left arm or the right arm of the robotic system gets close to any $\mathcal{O}_{j}$, then $V_{\text {obs }}$ increases.

To speed up the computation of $V(\boldsymbol{q})$, the distances between the robotic arms and the obstacles (i.e. $\mathrm{d}\left(\mathcal{L}_{\boldsymbol{q}}, \mathcal{R}_{\boldsymbol{q}}\right), \mathrm{d}\left(\mathcal{L}_{\boldsymbol{q}}, \mathcal{O}_{j}\right)$ and $\left.\mathrm{d}\left(\mathcal{R}_{\boldsymbol{q}}, \mathcal{O}_{j}\right)\right)$ are computed using a simplified model of the robot and the obstacles based on planes, spheres and capsules).

Note that as opposed to the potential-field function presented in [13], which was valid only for the motion planning of a free-flying robot with only translation DOFs, in this work the potential field has been extended to scenarios with a dual-arm robotic system (with more and different DOF types), and it could be extended easily to problems with several dual-arm mobile manipulators (i.e. parallel articulated systems with many DOFs of any type).

\section{B. The HD-RRT* Planner}

The proposed planning algorithm, called Human Demonstrated RRT* (HD-RRT*), is based on the RRT* planner [11] that has as parameters the sampling bias $\alpha$ towards the goal configuration and the advance step $\epsilon$ used in the extend function to grow the tree.

In order to cope with the limitations of the standard RRT* for high-dimensional configuration spaces, a modified version was proposed with the following changes [46]:

- A sampling bias: Once a solution has been found, the sampling is biased, with a given probability $\beta$, towards configurations around it. This guides the paths towards local optimal solutions.

- A node-rejection criteria: Those samples that may not be useful in finding a better solution than the current one are discarded. This keeps the tree as reduced as possible, thus reducing the computational cost.

The HD-RRT* planner proposed here introduces the next additional changes with respect to the modified RRT*:

- The optimization function: The minimization of this function, computed using Eq. (1)-(6), guides the solution towards short paths that follow as much as possible the demonstrated movements and that move away from obstacles and from self-collisions.

- The extension procedure: In the standard RRT* growth of the tree, the selected node is steered towards the sampled configuration. Here, this is modified to steer the node with a probability $\gamma$ towards low-cost directions (with a stochastic gradient-descent method) using the function CSTEER detailed below.

The proposed HD-RRT* planning algorithm uses the procedure CSTEER to extend the tree from a given configuration $\boldsymbol{q}_{\text {near }}$. The pseudocode and the flowchart of CSTEER are shown in Fig. 3, where the following functions are used: 

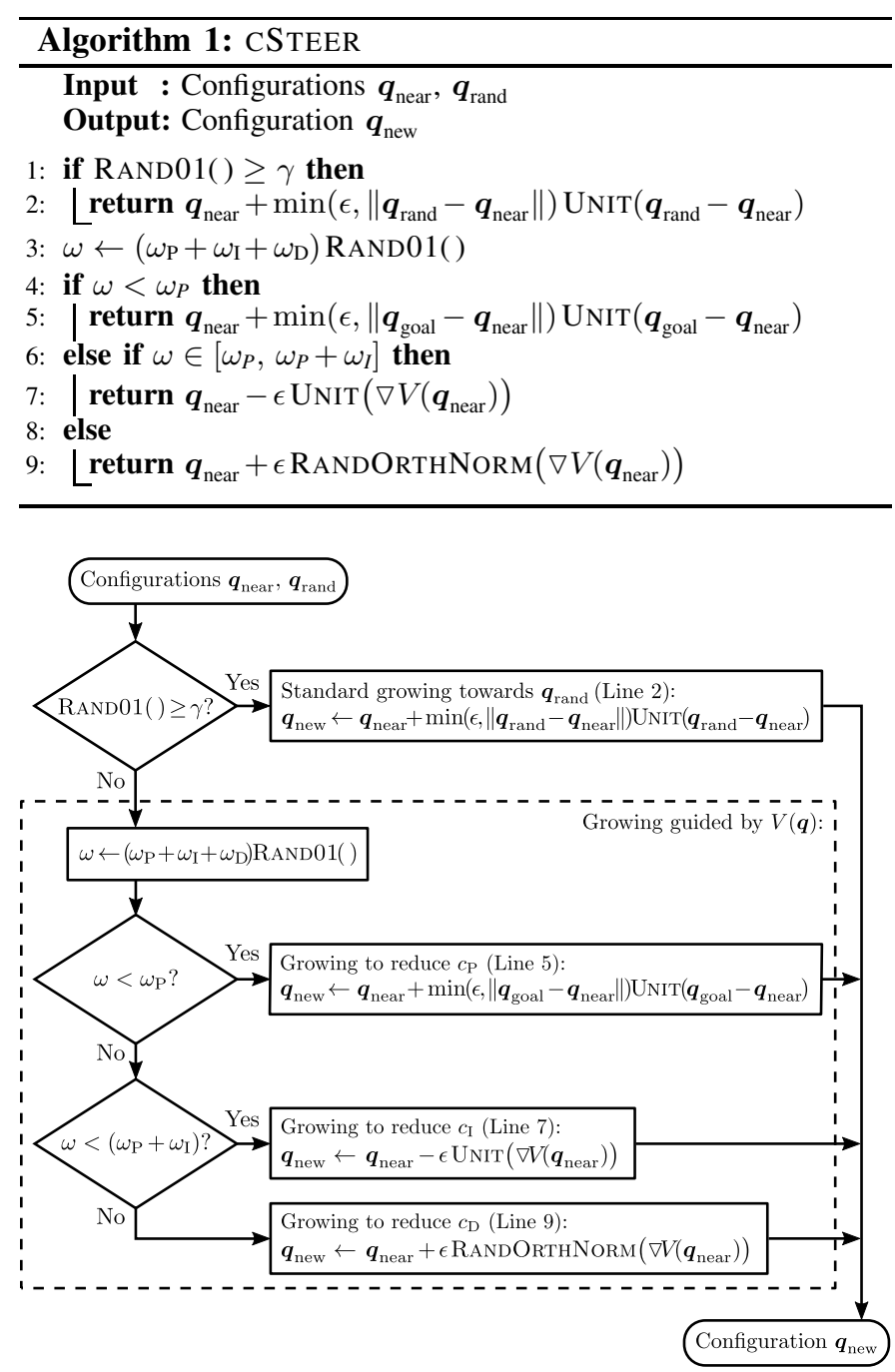

Fig. 3. Pseudocode and flowchart of the CSTEER algorithm.

- RAND01() returns a value uniformly chosen at random from the interval $[0,1]$.

- $\operatorname{Unit}(\boldsymbol{v})$ returns $\frac{\boldsymbol{v}}{\|\boldsymbol{v}\|}$ if $\|\boldsymbol{v}\| \neq 0$, and $\boldsymbol{v}$ otherwise.

- RANDORTHNORM $(\boldsymbol{v})$ returns a random, unitary vector orthogonal to $\boldsymbol{v}$ if $\|\boldsymbol{v}\| \neq 0$, and $\boldsymbol{v}$ otherwise.

- $V(\boldsymbol{q})$ is the potential-field value computed using Eq. (2).

- $\nabla V\left(\boldsymbol{q}_{\text {near }}\right)$ denotes the gradient of $V(\boldsymbol{q})$ evaluated at $\boldsymbol{q}_{\text {near }}$.

The extension is performed towards $\boldsymbol{q}_{\text {rand }}$ with an incremental step $\epsilon$ (Line 2), as it is done in the standard RRT algorithm. However, with a probability $\gamma<1$, a stochastic gradient-descent method minimizing the motion cost is applied instead. Note that the gradient-descent method can be trapped in local minima of the motion cost. Nevertheless, since the gradient descent is not applied always in all the iterations, the RRT* exploration properties are preserved and the possible local minimum traps are avoided (assuming $\gamma<1$ ). The extension direction is chosen randomly (Line 3 ) between the directions that minimize each component of the motion cost, i.e. $c_{\mathrm{P}}, c_{\mathrm{I}}$ and $c_{\mathrm{D}}$ (see Eq. (1)). Each of these cost components is chosen to be minimized with a probability proportional to its weight value $\omega_{\mathrm{P}}, \omega_{\mathrm{I}}$ and $\omega_{\mathrm{D}}$ respectively (e.g. the greater

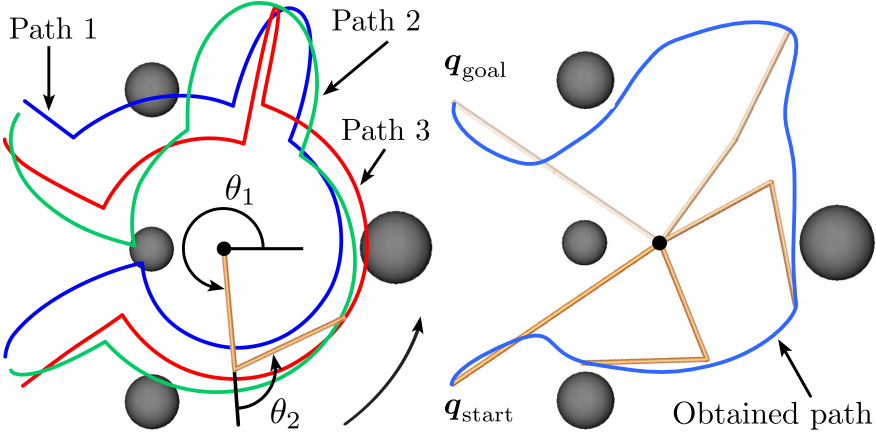

Fig. 4. 2-link planar manipulator problem: demonstrated paths (left) and obtained solution path (right).

$\omega_{\mathrm{P}}$ regarding $\omega_{\mathrm{I}}$ and $\omega_{\mathrm{D}}$, the greater the probability that the tree grows in the direction that minimizes $c_{\mathrm{P}}$ ). Then:

- Since $c_{\mathrm{P}}$ measures the path length, the direction pointing from $\boldsymbol{q}_{\text {near }}$ towards $\boldsymbol{q}_{\text {goal }}$ minimizes $c_{\mathrm{P}}$ (Line 5).

- Since $c_{\mathrm{I}}$ measures the average value of the potential field along the path, and the gradient $\nabla V(\boldsymbol{q})$ of the potential field points in the direction of the local greatest growth of $V(\boldsymbol{q})$, then the direction that minimizes $c_{\mathrm{I}}$ points in the opposite direction of $\nabla V(\boldsymbol{q})$, i.e. the direction in which $V(\boldsymbol{q})$ locally decreases (Line 7).

- Since $c_{\mathrm{D}}$ measures the variations of $V(\boldsymbol{q})$ along the motion, then any direction orthogonal to $\nabla V(\boldsymbol{q})$ minimizes $c_{\mathrm{D}}$ because $V(\boldsymbol{q})$ does not locally grow in any direction perpendicular to $\nabla V(\boldsymbol{q})$ (Line 9).

After testing for different tasks, the parameters of the HD-RRT* algorithm have been empirically set to $\alpha=0.05$, $\beta=0.1$, and $\gamma=0.1$, being $\epsilon$ dependant on the problem. Regarding the motion-cost function, the motion connecting straightly $\boldsymbol{q}_{\text {start }}$ and $\boldsymbol{q}_{\text {goal }}$ has been used to set each weight of the motion-cost function: $\omega_{\Gamma}=c_{\Gamma}\left(\boldsymbol{q}_{\text {start }}, \boldsymbol{q}_{\text {goal }}\right)^{-1}$ for $\Gamma \in\{\mathrm{P}, \mathrm{I}, \mathrm{D}\}$ (see Eq. (1)). Besides, the parameters of the potential fields have been empirically set to: $\lambda_{\text {goal }}=0.1$, $\sigma_{\text {goal }}=0.1 ; \lambda_{i}=0.1, \sigma_{i}=7 \forall i ; \lambda_{\text {arms }}=0.3, \sigma_{\text {arms }}=10$; and $\lambda_{j}=0.3, \sigma_{j}=10 \forall j$ (see Eq. (3)-(6) ). In fact, the same values of the parameters are used in the conceptual and the real examples presented in the next subsection, with the exception of the parameter $\epsilon$ that is the unique task-dependant parameter. The sensibility of the system performance with respect to the planner parameters is not high, thus determining them is not a critical issue.

\section{Validation and performance analysis}

The approach has been implemented within The Kautham Project [47], a motion planning and simulation framework for teaching and research. The experiments described below were obtained running the planner in a $2.13-\mathrm{GHz}$ Intel 2, 4-GB RAM PC.

First, for illustrative purposes, a simple example has been set up. It consists of a $2 \mathrm{D}$ scenario where a 2-link planar manipulator must go from the start configuration $\boldsymbol{q}_{\text {start }}$ to the goal configuration $\boldsymbol{q}_{\text {goal }}$ avoiding collisions with circular obstacles (see Fig. (4). Three demonstration paths and $\boldsymbol{q}_{\text {goal }}$ 

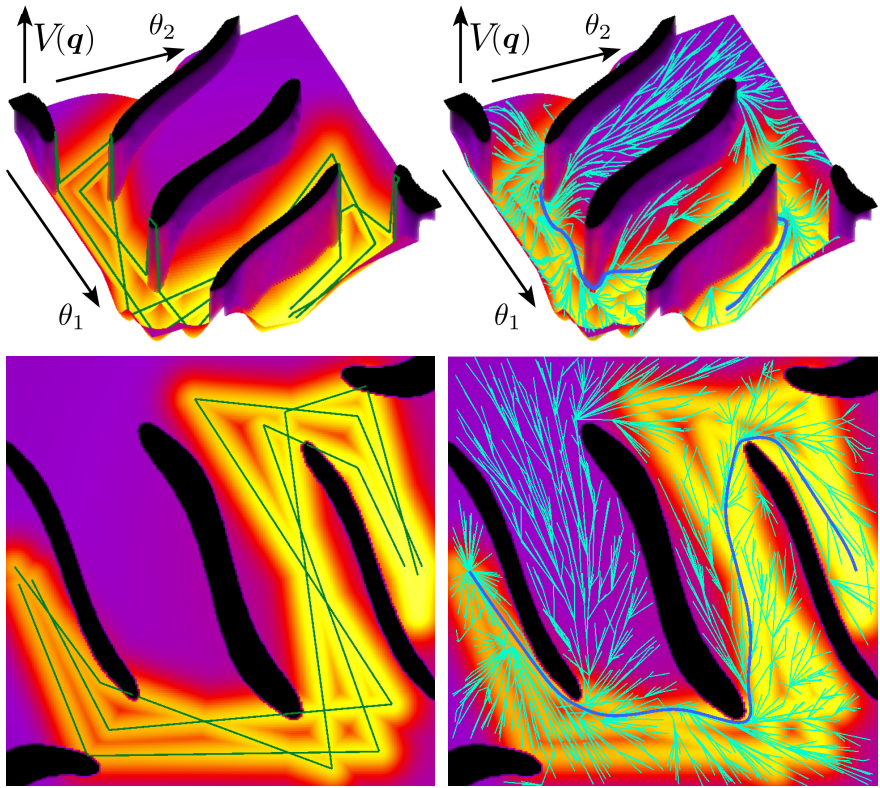

Fig. 5. Tridimensional and top views of the potential field $V(\boldsymbol{q})$ over the configuration space $\mathcal{C}$ for the example in Fig. 4 (brighter colors represent lower values of $V(\boldsymbol{q})$ ), with the demonstrated paths (left), and the obtained solution path and sample tree (right).

were used to generate the attractive potential field, while the circular obstacles generate repulsive potential fields. The combination of these attractive and repulsive potential fields forms the potential-field function $V(\boldsymbol{q})$. Fig. 44-left shows the three demonstration paths in the problem space and Fig. 4 . right shows the obtained solution. Fig. 5 shows resulting potential-field function $V(\boldsymbol{q})$ in the configuration space, including the three demonstration paths in Fig. 5-left and the resulting tree of samples and the obtained solution Fig. 5-right.

Note that $V(\boldsymbol{q})$ is shaped like a plateau in the regions of $\mathcal{C}$ where the manipulator is in collision with the obstacles (depicted in black in Fig. 5), while, on the other hand, the demonstration paths originate valleys (bright colored in Fig. 55). Therefore, the use of $V(\boldsymbol{q})$ in the $c_{\mathrm{I}}$ and $c_{\mathrm{D}}$ cost components in Eq. (1) enforce the solution path to follow the demonstrations as close as possible, while $c_{\mathrm{P}}$ tries to shorten the path.

The planning procedure assures that a solution path avoiding obstacles and self-collisions is found (if one exists) due to the asymptotic completeness of any RRT-based planning algorithm, even if the demonstration paths are not collision-free (as it actually happens in this example, see Fig. 4 and Fig. 5). It must be also remarked that the fact that the demonstration paths provides relevant information on a given task is more significant than the number of demonstration paths used, and that the method works well even with a single demonstration (in this case the valleys are sharper, and they become wider when there are several different demonstration paths, with the valley width growing when the dispersion of the demonstration paths grows). On the other hand, the computation time of the motion-cost function grows when the number of demonstration paths increases (as it is expected according to Eq. (4), but it does not produce any other negative consequence.

Numerical results of the 2-link planar manipulation problem
TABLE I

AVERAGE RESULTS OF THE CONCEPTUAL EXAMPLE MOTION PLANNING.

\begin{tabular}{|c|c|c|c|}
\hline Time first solution & Path length & Total cost $c_{\mathcal{P}}$ & Unit cost $\hat{c}_{\mathcal{P}}$ \\
\hline $0.26 \mathrm{~s}$ & $10.825 \mathrm{rad}$ & 1.195 & 0.111 \\
\hline
\end{tabular}

TABLE II

AVERAGE RESULTS OF THE ASSEMBLY EXAMPLE MOTION PLANNING.

\begin{tabular}{|l|c|c|}
\hline Planning space / Dimension & $\mathcal{C} / 12$ & $B_{k} / 2$ \\
\hline \hline Used time $[\mathrm{s}]$ & 100 & 10 \\
\hline Time first solution $[\mathrm{s}]$ & 1.35 & 0.33 \\
\hline Solution length $[\mathrm{rad}]$ & 5.513 & 5.141 \\
\hline Total cost $c_{\mathcal{P}}$ & 1.095 & 1.186 \\
\hline Unit cost $\hat{c}_{\mathcal{P}}$ & 0.199 & 0.231 \\
\hline Tree growing success [\%] & 73.49 & 94.56 \\
\hline
\end{tabular}

using three demonstration paths are summarized in Table [ where the average values after 100 executions are shown (remind that the sampling-based planners rely on a random process and therefore generate a different solution each execution). A maximum time of $10 \mathrm{~s}$ was allowed for each execution, this was enough to get a $100 \%$ of success rate, i.e. the system finds always a collision-free path avoiding self-collisions and collisions with the obstacles. The collected data include:

- The final solution length $L$ (measured in $\mathcal{C}$ as the summation of joint movements in radians).

- The final path cost $c_{\mathcal{P}}$ (defined as the sum of the motion costs of all the segments that form the path).

- The unit path cost $\hat{c}_{\mathcal{P}}$ (computed as $c_{\mathcal{P}}$ divided by $L$ ).

After this simple example, the planning of the motions for an assembly task is used as a real example of the proposed planning procedure (see Fig. 11). This task consists in holding a cylindrical box with one hand and a soda can with the other, and then move both objects to a pre-assembly pose that allows the insertion of the can into the box. Note that the start and the goal dual-arm configurations are given and that the proposed algorithm plans the path of the whole dual-arm robotic system. For this example, the movements of a human operator were captured while solving the task (see Fig. 1), and then these movements were mapped to the dual-arm system (see Subsection (III-A). These human movements were used as demonstration paths to generate the attractive potential fields as well as to obtain the zero-order basis ${ }^{0} S$ of the demonstrated task. The axes of ${ }^{0} S$ are sorted in decreasing order of the associated sample dispersions, then the subspace $B_{k}$ spanned by the first $k$ axes has a dimension lower than the complete $\mathcal{C}$ space and, at the same time, contains a high percentage of the sample variance. In this work, $k$ has been chosen so that the accumulated sample variance of the first $k$ axes surpasses the $95 \%$ of the total sample variance, i.e. $k$ depends on the human demonstrations of the task and it may vary from task to task. Following this criterion, for this example, only two axes were needed (the bottom-right picture of Fig. 11 shows the distribution of the accumulated sample variance of the captured motion as a function of the number of synergies). The potential field was generated using five demonstration paths, and the 


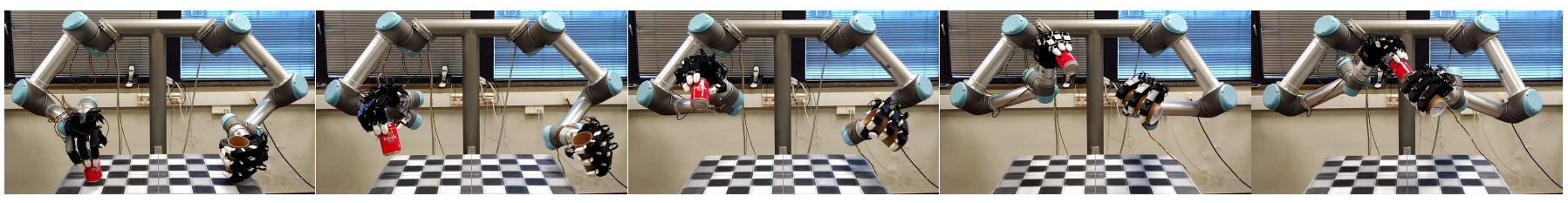

Fig. 6. Snapshots of a solution path for the assembly example planned in the reduced subspace $B_{k}$.

planning of the robot motions has been done using the whole configuration space $\mathcal{C}$ and also using the reduced planning subspace $B_{k}$, with maximum allowed planning times of $100 \mathrm{~s}$ and $10 \mathrm{~s}$, respectively, assuring a $100 \%$ of success rate.

Fig. 6 shows snapshots of an instance of the solution path for the assembly task obtained using the reduced subspace $B_{k}$. Videos of each step of the experiment for the assembly task are available in [48]. Table [1 shows the average results obtained after 100 executions for each case using five demonstration paths. The table includes, as tree growing success, the percentage of times that the CSTEER function returned a collision-free motion (i.e. the percentage of iterations in which no collisions occur and the tree actually grows).

As expected, the best paths (the ones with the lowest cost) are obtained when the whole $\mathcal{C}$ space was used. Nevertheless, planning in $B_{k}$ allows a much shorter planning time (due to the reduced dimension of the subspace) without incrementing excessively the cost of the path. In addition, the use of $B_{k}$ increases the probability of obtaining collision-free configurations (see the tree growing success in Table III) because fewer self-collisions occur, and therefore the efficiency of the planning procedure increases.

\section{HUMAN-LIKENESS EVALUATION}

\section{A. Definition of a human-likeness index}

The proposed planning algorithm tries to mimic the human demonstrations (which does not mean "following a specific human path") as long as it does not imply that the arms are dangerously close to each other or to the obstacles. In addition, the goal configuration acts as an attractive point for the planned path. Therefore, the planned path does not follow strictly the human demonstrated motion and then the human-likeness of the planned path may be somehow spoiled. In order to evaluate the human-likeness of different paths (obtained with the proposed planning procedure or with any other), this paper introduces a human-likeness index $\mathcal{Q}_{\mathcal{P}}$. This index computes the misalignment of a path with respect to the first-order bases ${ }^{1} S$ obtained from human movements. Since as detailed in Section III-B, the configuration space is split into cells, each one having an associated ${ }^{1} S$ basis, the value $\mathcal{Q}_{\mathcal{P}}$ of a path $\mathcal{P}$ is computed as

$$
\mathcal{Q}_{\mathcal{P}}=1-\frac{1}{L} \int_{\mathcal{P}} \operatorname{Mis} \text { ALIGNMENT }(\boldsymbol{q}, \boldsymbol{v}) \mathrm{d} \boldsymbol{q}
$$

where $L$ is the path length, $\boldsymbol{v}=\dot{\boldsymbol{q}}$, and $\operatorname{Misalignment}(\boldsymbol{q}, \boldsymbol{v})$ is the function that returns the misalignment $\eta$ of the direction $\boldsymbol{v}$ with respect to the basis ${ }^{1} S(\boldsymbol{\mu}, \Sigma)$ of the cell where $\boldsymbol{q}$ lies. This misalignment $\eta$ is measured as

$$
\eta=\frac{1}{\pi} \operatorname{acos}\left((1-\rho) \Phi_{\boldsymbol{\mu}}+\rho \Phi_{\Sigma}\right)
$$

where:

- $\rho \in[0,1]$ is a weighting variable that represents the proximity of the basis ${ }^{1} S(\boldsymbol{\mu}, \Sigma)$ to the origin of the velocity space, i.e. $\rho$ increases as the origin of ${ }^{1} S$ gets closer to the origin of the velocity space.

$\rho$ is computed as two times the probability $\mathrm{P}$ that a random vector $\boldsymbol{x}$ obtained from the normal multivariate distribution $\mathcal{N}(\boldsymbol{\mu}, \Sigma)$ (i.e. with barycenter $\boldsymbol{\mu}$ and covariance matrix $\Sigma$ ) satisfies $\boldsymbol{\mu} \cdot \boldsymbol{x}<0$. The probability $\mathrm{P}$ is given by:

$$
\mathrm{P}(\boldsymbol{\mu} \cdot \boldsymbol{x}<0 \mid \boldsymbol{x} \sim \mathcal{N}(\boldsymbol{\mu}, \Sigma))=\frac{1}{2}-\frac{1}{2} \operatorname{erf}\left(\frac{\boldsymbol{\mu} \cdot \boldsymbol{\mu}}{\sqrt{2 \boldsymbol{\mu}^{\top} \Sigma \boldsymbol{\mu}}}\right)
$$

where $\operatorname{erf}(x)$ is the error function. Then,

$$
\rho=1-\operatorname{erf}\left(\frac{\boldsymbol{\mu} \cdot \boldsymbol{\mu}}{\sqrt{2 \boldsymbol{\mu}^{\top} \Sigma \boldsymbol{\mu}}}\right)
$$

Therefore, when the first-order basis ${ }^{1} S$ is exactly centered at the origin (i.e. $\|\boldsymbol{\mu}\|=0$ ), half of the distribution $\mathcal{N}$ satisfies the inequality and, hence, $\rho=1$. As the first-order basis ${ }^{1} S$ gets away from the origin (i.e. $\|\boldsymbol{\mu}\| \rightarrow \infty$ ), only a reduced region of $\mathcal{N}$ satisfies the inequality and, therefore, $\rho \rightarrow 0$.

- $\Phi_{\mu} \in[-1,1]$ is a measure that represents the alignment between $\boldsymbol{v}$ and $\boldsymbol{\mu}$ (see Fig. 7fleft).

$\Phi_{\mu}$ is computed as

$$
\Phi_{\boldsymbol{\mu}}=\operatorname{sgn}(\boldsymbol{v} \cdot \boldsymbol{\mu}) e^{-\frac{1}{2}(\boldsymbol{w}-\boldsymbol{\mu})^{\top} \Sigma^{-1}(\boldsymbol{w}-\boldsymbol{\mu})}
$$

where $\operatorname{sgn}(x)$ is the sign function. $\Phi_{\mu}$ is positive if $(\boldsymbol{v} \cdot \boldsymbol{\mu})>0$, and negative otherwise. Besides, $\left|\Phi_{\boldsymbol{\mu}}\right|$ is proportional to the value of the probability density function of $\mathcal{N}(\boldsymbol{\mu}, \Sigma)$ evaluated at $\boldsymbol{w}$, which is a scaled version of $\boldsymbol{v}$ so that the projection of $\boldsymbol{w}$ into $\boldsymbol{\mu}$ is $\boldsymbol{\mu}$ itself (see Fig. 7.left), i.e. $\boldsymbol{w}=\frac{\boldsymbol{\mu} \cdot \boldsymbol{\mu}}{\boldsymbol{v} \cdot \boldsymbol{\mu}} \boldsymbol{v}$. Therefore, $\left|\Phi_{\boldsymbol{\mu}}\right|=1$ when $\boldsymbol{v}$ and $\boldsymbol{\mu}$ are parallel and $\left|\Phi_{\boldsymbol{\mu}}\right|=0$ when $\boldsymbol{v}$ and $\boldsymbol{\mu}$ are orthogonal.

$\Phi_{\Sigma} \in[-1,1]$ is a measure that represents the alignment of $\boldsymbol{v}$ and the direction $\boldsymbol{u}_{1}$ of largest variance of $\Sigma$ (see Fig. 7 right).

$\Phi_{\Sigma}$ is computed as

$$
\Phi_{\Sigma}=2 \frac{\hat{\boldsymbol{v}}^{\top} \Sigma \hat{\boldsymbol{v}}}{\boldsymbol{u}_{1}^{\top} \Sigma \boldsymbol{u}_{1}}-1 \quad \text { with } \quad \hat{\boldsymbol{v}}=\frac{\boldsymbol{v}}{\|\boldsymbol{v}\|}
$$

where $\hat{\boldsymbol{v}}^{\top} \Sigma \hat{\boldsymbol{v}}$ is the variance of $\Sigma$ in the direction of $\boldsymbol{v}$, and $\boldsymbol{u}_{1}^{\top} \Sigma \boldsymbol{u}_{1}$ is the variance of $\Sigma$ in the direction of $\boldsymbol{u}_{1}$. The quotient of these two variances takes the maximum value 1 when $\boldsymbol{v}$ and $\boldsymbol{u}_{1}$ are parallel, and the minimum value 0 when $\boldsymbol{v}$ is parallel to $\boldsymbol{u}_{m}$, the direction of smallest variance of $\Sigma$. To obtain $\Phi_{\Sigma}$, this quotient is then 

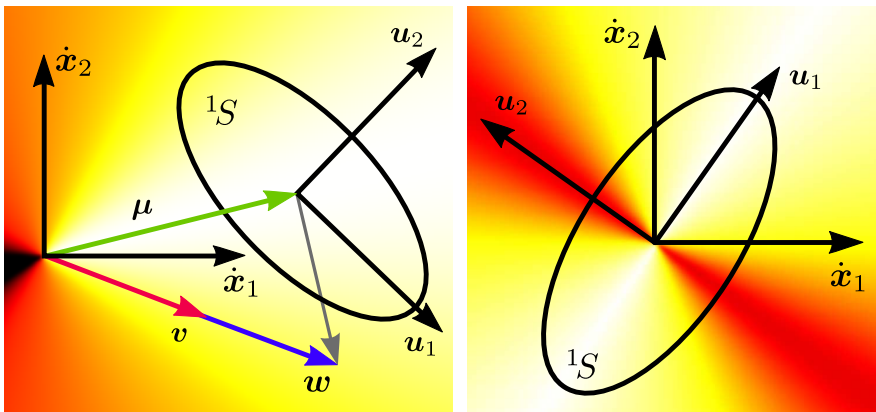

Fig. 7. Misalignment of each direction $\boldsymbol{v}=\left[\dot{x}_{1}, \dot{x}_{2}\right]$, when the first-order basis ${ }^{1} S(\boldsymbol{\mu}, \Sigma)$ is far from the origin, i.e. $\Phi \approx \Phi_{\boldsymbol{\mu}}$ (left), and when ${ }^{1} S$ is exactly centered at the origin, i.e. $\Phi=\Phi_{\Sigma}$ (right). Brighter colors denote better alignments. $\Sigma$ is represented by an ellipse oriented according to the eigenvectors $\boldsymbol{u}_{i}$ of $\Sigma$ and with semiaxes proportional to the square roots of the eigenvalues of $\Sigma$. A sample of the velocities $\boldsymbol{v}$ and $\boldsymbol{w}$ is also shown.

expanded from the interval $[0,1]$ to the interval $[-1,1]$ with a linear transformation.

Note that the misalignment value $\eta \in[0,1]$ and it is small when the advance direction $\boldsymbol{v}$ is similar to the synergy directions. When the difference between $\boldsymbol{v}$ and the synergy directions increases, the misalignment increases.

The pseudocode and the flowchart of the Misalignment function are shown in Fig. 8 First, the first-order synergy basis ${ }^{1} S(\boldsymbol{\mu}, \Sigma)$ of the cell where $\boldsymbol{q}$ lies is obtained with the function $\operatorname{FoSBASIS}(\boldsymbol{q})$ (Line 1) which returns $\emptyset$ if no first-order basis is available, i.e. $\boldsymbol{q}$ is outside $B\left({ }^{0} S_{G}\right)$ and therefore it does not belong to any cell (see Subsection III-B). If ${ }^{1} S=\emptyset$ the misalignment $\eta$ is set to the maximum value 1 (Line 2); otherwise, $\rho$ is computed following Eq. (10) (Line 4), $\Phi_{\mu}$ is computed according to Eq. (11) (Line 5), and $\Phi_{\Sigma}$ is computed according to Eq. (12) (Line 6). Finally, $\eta$ is computed according to Eq. (8) (Line 7).

Now, since the path $\mathcal{P}$ is composed of a sequence of $n$ consecutive configurations $\boldsymbol{q}_{i}$ connected by rectilinear motions, $\mathcal{Q}_{\mathcal{P}}$ from Eq. (7) can be approximated as

$$
\mathcal{Q}_{\mathcal{P}} \approx 1-\sum_{i=1}^{n-1} \operatorname{Misalignment}\left(\boldsymbol{q}_{i}, \boldsymbol{q}_{i+1}-\boldsymbol{q}_{i}\right) \frac{\left\|\boldsymbol{q}_{i+1}-\boldsymbol{q}_{i}\right\|}{L}
$$

Therefore a path with a high $\mathcal{Q}_{\mathcal{P}}$ value, is highly aligned in $\mathcal{C}$ with the human movements. Then, if the robot kinematic structure is anthropomorphic (and the similar to the human operator, the better), the position and velocity of the robot wrists and the human wrists are similar.

The human-likeness index $\mathcal{Q}_{\mathcal{P}}$ depends on the ${ }^{1} S$ bases used. $\mathcal{Q}_{\mathcal{P}}$ can be tailored to any given particular task by using the corresponding ${ }^{1} S$ bases, and used for the evaluation of the human-likeness of the execution of that particular task. Note that the ${ }^{1} S$ bases depend on the mapping of the human movements to the robot configuration space, thus a mapping preserving the human-likeness should be used to make ${ }^{1} S$ really represent the human-like movements.

In this paper, we propose the use of ${ }^{1} S$ obtained from natural free-movements of the operator while he/she freely moves both arms and hands in an unconstrained way (i.e. without performing any specific task) trying to cover the whole workspace
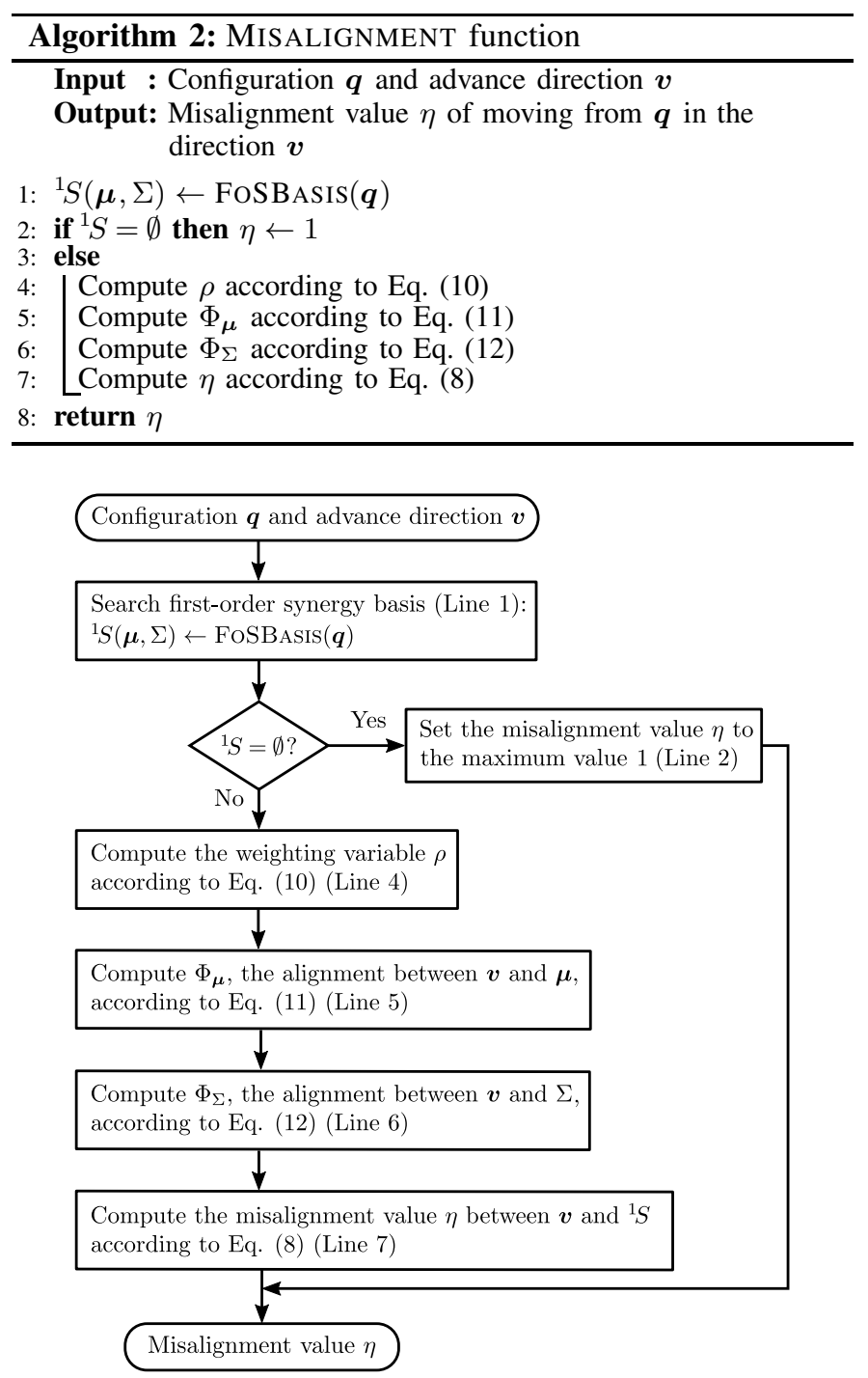

Fig. 8. Pseudocode and flowchart of the Misalignment function.

in front of the body. There is no guarantee that the operator actually covers the whole workspace, but it is expected that he/she performs his/her most natural and evident movements.

\section{B. Evaluation}

Zero- and first-order synergy bases were computed using the free-movements described above. The configuration space $\mathcal{C}$ was split into 64 cells based on the synergy differences, i.e. a first-order basis ${ }^{1} S$ was assigned to each cell of $\mathcal{C}$. These bases were used for the computation of $\mathcal{Q}_{\mathcal{P}}$, which was applied to the evaluation of the human-likeness of the solutions found for the assembly example defined in Section $\amalg$ II-A using:

a) The HD-RRT* planner with several demonstrations, planning in the whole configuration space $\mathcal{C}$.

b) The HD-RRT* planner with several demonstrations, planning in the lower-dimensional subspace $B_{k}$.

c) The planner introduced in [35], that simply computes zero-order synergies for different tasks and uses them to reduce the dimension of the search space, thus reducing the computational cost. 
TABLE III

AVERAGE HUMAN-LIKENESS VALUE OF THE ASSEMBLY EXAMPLE USING THE PRESENTED APPROACH WITH SEVERAL DEMONSTRATIONS $(a, b)$, A SIMILAR APPROACH (c), THE RRT (d, e) AND AN ARTIFICIAL PATH (f).

\begin{tabular}{|l||l|l|l|l|}
\hline Case & Planning dim. & Used time [s] & Path length [rad] & Quality $\mathcal{Q}_{\mathcal{P}}$ \\
\hline
\end{tabular}

\begin{tabular}{|c||c|c|c|c|}
\hline \hline a) & 12 & 100 & 5.513 & 0.594 \\
\hline b) & 2 & 10 & 5.141 & 0.573 \\
\hline c) & 4 & 0.35 & 4.990 & 0.465 \\
\hline d) & 12 & 32.39 & 6.647 & 0.433 \\
\hline e) & 2 & 18.38 & 5.965 & 0.428 \\
\hline f) & - & - & 17.452 & 0.045 \\
\hline
\end{tabular}

d) The standard RRT planner [6], planning in the whole configuration space $\mathcal{C}$.

e) The standard RRT planner, planning in the lowerdimensional subspace $B_{k}$.

f) Two rectilinear segments in $\mathcal{C}$ connecting the start and the goal configurations through an empirically selected configuration $\boldsymbol{q}_{\mathrm{m}} \notin B\left({ }^{0} S_{G}\right)$, i.e. $\boldsymbol{q}_{\mathrm{m}}$ is not in the subspace of the sampled configurations of the free-movements. Note that in this case no motion planning is performed but even so the path is checked to be free of collisions, either involving the obstacles or both robotic arms.

Table III shows the average results obtained, for each case, after 100 executions. It can be noted that the proposed planner obtains paths with a significant better $\mathcal{Q}_{\mathcal{P}}$ quality (even though the presented approach needs a longer planning time), i.e. the proposed procedure finds solution paths that are better aligned with the natural movements of the human operator and that therefore are more human-like. Note that the approach presented in this work obtains a better $\mathcal{Q}_{\mathcal{P}}$ even though the human movements used in the motion planning are different to the ones used to compute $\mathcal{Q}_{\mathcal{P}}$. The path with the greatest quality is obtained when the motions are planned in the whole $\mathcal{C}$ space. However, the use of the subspace $B_{k}$ is the best option since it reduces significantly the computational cost without penalizing considerably $\mathcal{Q}_{\mathcal{P}}$. Case (c) is very fast since it is not based in any optimization method and hence ends as soon as a solution is found, and has a relative $\operatorname{good} \mathcal{Q}_{\mathcal{P}}$. The poorest quality is obtained with the manually-set path, denoting that this path is not much human-like. The planner used in cases (d) and (e) does not consider human-likeness nor path length as a quality index. Hence, bad results are obtained for both measures.

Videos of paths obtained for the assembly task with the considered approaches are available in [48].

\section{DISCUSSION AND FUTURE WORK}

This paper has introduced a motion planning procedure, designed for anthropomorphic dual-arm robotic systems, that allows to solve manipulation tasks in a human-like fashion. To this end, the movements of a human operator have been used to generate attractive potential fields over the configuration space. The motion planning has been solved with an RRT*-based algorithm, with a stochastic gradient descent method to minimize a motion cost. The algorithm navigates through the potential fields and biases the tree growth towards the human-like movements. In addition, the synergies (couplings between DOFs) of the demonstration movements have been computed to find a lower-dimensional subspace where the motion planning can be solved more efficiently, basically due to the fact that the sampling procedure produces in fewer self-collision configurations. The proposed approach has been illustrated with a conceptual example and a real example executed with a physical dual-arm robotic system. A human-likeness index, based on first-order synergies (correlations between joint velocities) obtained from human movements, has been also proposed and used for comparisons. The obtained results show that the proposed procedure obtains paths that are more human-like.

As a conclusion, this paper has presented a simple yet efficient way to compute paths for dual-arm robotic systems with human-like appearance, and it opens interesting potential research lines, such as the use of demonstration paths in the joint-velocity space and their first-order synergies during the motion planning. Another interesting research direction concerns the optimization of the presented human-likeness index while solving the motion planning in order to better mimic human task executions.

\section{REFERENCES}

[1] B. Tovar, R. Murrieta-Cid, and S. LaValle, "Distance-Optimal navigation in an unknown environment without sensing distances," IEEE Trans. Robotics, vol. 23, no. 3, pp. 506-518, June 2007.

[2] H. Wang, Y. Chen, and P. Soueres, "A geometric algorithm to compute time-optimal trajectories for a bidirectional steered robot," IEEE Trans. Robotics, vol. 25, no. 2, pp. 399-413, Apr. 2009.

[3] T. Fukuda, R. Michelini, V. Potkonjak, S. Tzafestas, K. Valavanis, and M. Vukobratovic, "How far away is "artificial man"," IEEE Robotics and Automation Mag., vol. 8, no. 1, pp. 66-73, Mar. 2001.

[4] M. Elbanhawi and M. Simic, "Sampling-based robot motion planning: A review," IEEE Access, vol. 2, pp. 56-77, Jan. 2014.

[5] L. Kavraki, P. Svestka, J.-C. Latombe, and M. Overmars, "Probabilistic roadmaps for path planning in high-dimensional configuration spaces," IEEE Trans. Robotics and Automation, vol. 12, no. 4, pp. 566-580, Aug. 1996.

[6] J. Kuffner and S. LaValle, "RRT-Connect: An efficient approach to single-query path planning," in Proc. IEEE Int. Conf. Robotics and Automation, Apr. 2000, pp. 995-1001.

[7] M. Stilman, "Global manipulation planning in robot joint space with task constraints," IEEE Trans. Robotics, vol. 26, no. 3, pp. 576-584, June 2010.

[8] L. Jaillet, J. Cortés, and T. Siméon, "Sampling-based path planning on configuration-space costmaps," IEEE Trans. Robotics, vol. 26, no. 4, pp. 635-646, Aug. 2010.

[9] J. Lee, O. Kwon, L. Zhang, and S. Yoon, "A selective retraction-based RRT planner for various environments," IEEE Trans. Robotics, vol. 30, no. 4, pp. 1002-1011, Aug. 2014.

[10] J. Rosell, R. Suárez, and A. Pérez, "Path planning for grasping operations using an adaptive PCA-based sampling method," Autonomous Robots, vol. 35, no. 1, pp. 27-36, July 2013.

[11] S. Karaman and E. Frazzoli, "Sampling-based algorithms for optimal motion planning," Int. J. Robotics Research, vol. 30, no. 7, pp. 846894, June 2011.

[12] A. Qureshi, K. Iqbal, S. Qamar, F. Islam, Y. Ayaz, and N. Muhammad, "Potential guided directional-RRT* for accelerated motion planning in cluttered environments," in Proc. IEEE Int. Conf. Mechatronics and Automation, Aug. 2013, pp. 519-524.

[13] N. García, R. Suárez, and J. Rosell, "HG-RRT*: Human-Guided optimal random trees for motion planning," in Proc. IEEE Int. Conf. Emerging Technologies and Factory Automation, Sept. 2015.

[14] S. M. Khansari-Zadeh and O. Khatib, "Learning potential functions from human demonstrations with encapsulated dynamic and compliant behaviors," Autonomous Robots, vol. 41, no. 1, pp. 45-69, Jan. 2017. 
[15] S. Cho and S. Jo, "Incremental online learning of robot behaviors from selected multiple kinesthetic teaching trials," IEEE Trans. Systems, Man and Cybernetics: Systems, vol. 43, no. 3, pp. 730-740, May 2013.

[16] N. Fligge, J. McIntyre, and P. van der Smagt, "Minimum jerk for human catching movements in 3d," in IEEE RAS/EMBS Int. Conf. Biomedical Robotics and Biomechatronics, 2012, pp. 581-586.

[17] A. Piazzi and A. Visioli, "Global minimum-jerk trajectory planning of robot manipulators," IEEE Trans. Industrial Electronics, vol. 47, no. 1, pp. 140-149, Feb 2000

[18] M. Breteler, S. Gielen, and R. Meulenbroek, "Endpoint constraints in aiming movements: Effects of approach angle and speed," Biological Cybernetics, vol. 85, no. 1, pp. 65 - 75, July 2001.

[19] S. Albrecht, K. Ramirez-Amaro, F. Ruiz-Ugalde, D. Weikersdorfer, M. Leibold, M. Ulbrich, and M. Beetz, "Imitating human reaching motions using physically inspired optimization principles," in Proc. IEEE-RAS Int. Conf. Humanoid Robots, Oct. 2011, pp. 602-607.

[20] W. Park, D. B. Chaffin, and B. J. Martin, "Toward memory-based human motion simulation: Development and validation of a motion modification algorithm," IEEE Trans. Systems, Man and Cybernetics A: Systems and Humans, vol. 34, no. 3, pp. 376-386, May 2004.

[21] C. Rigotti, P. Cerveri, G. Andreoni, A. Pedotti, and G. Ferrigno, "Modeling and driving a reduced human mannequin through motion captured data: A neural network approach," IEEE Trans. Systems, Man and Cybernetics A: Systems and Humans, vol. 31, no. 3, pp. 187-193, May 2001.

[22] T. Tsuji, Y. Tanaka, and M. Kaneko, "Bio-mimetic trajectory generation based on human arm movements with a nonholonomic constraint," IEEE Trans. Systems, Man and Cybernetics A: Systems and Humans, vol. 32 no. 6, pp. 773-779, Nov. 2002

[23] Z. Zhang, Z. Li, Y. Zhang, Y. Luo, and Y. Li, "Neural-Dynamic-MethodBased dual-arm CMG scheme with time-varying constraints applied to humanoid robots," IEEE Trans. Neural Networks and Learning Systems, vol. 26, no. 12, pp. 3251-3262, Dec. 2015.

[24] Z. Li, K. Yang, S. Bogdan, and B. Xu, "On motion optimization of robotic manipulators with strong nonlinear dynamic coupling using support area level set algorithm," Int. J. Control, Automation and Systems, vol. 11, no. 6, pp. 1266-1275, July 2013.

[25] X. Ding and C. Fang, "A novel method of motion planning for an anthropomorphic arm based on movement primitives," IEEE/ASME Trans. Mechatronics, vol. 18, no. 2, pp. 624-636, Apr. 2013.

[26] J. Romero, T. Feix, C. Ek, H. Kjellström, and D. Kragic, "Extracting postural synergies for robotic grasping," IEEE Trans. Robotics, vol. 29, no. 6, pp. 1342-1352, Dec. 2013.

[27] M. Ciocarlie and P. Allen, "Hand posture subspaces for dexterous robotic grasping," Int. J. Robotics Research, vol. 28, no. 7, pp. 851-867, July 2009.

[28] N. Hogan and D. Sternad, "Dynamic primitives of motor behavior," Biological Cybernetics, vol. 106, no. 11-12, pp. 727-739, Dec. 2012

[29] J. Rosell and R. Suárez, "Using hand synergies as an optimality criterion for planning human-like motions for mechanical hands," in Proc. IEEERAS Int. Conf. Humanoid Robots, Nov. 2014, pp. 232-237.

[30] F. Ficuciello, G. Palli, C. Melchiorri, and B. Siciliano, "Postural synergies of the UB Hand IV for human-like grasping," Robotics and Auton. Systems, vol. 62, no. 4, pp. 515-527, Apr. 2014

[31] M. Gabiccini, A. Bicchi, D. Prattichizzo, and M. Malvezzi, "On the role of hand synergies in the optimal choice of grasping forces," Autonomous Robots, vol. 31, no. 2-3, pp. 235-252, Oct. 2011.

[32] G. Palli, C. Melchiorri, G. Vassura, U. Scarcia, L. Moriello, G. Berselli, A. Cavallo, G. D. Maria, C. Natale, S. Pirozzi, C. May, F. Ficuciello, and B. Siciliano, "DEXMART hand: Mechatronic design and experimental evaluation of synergy-based control for human-like grasping," Int. J. Robotics Research, vol. 33, no. 5, pp. 799-824, Apr. 2014.

[33] M. Gabiccini, A. Bicchi, D. Prattichizzo, and M. Malvezzi, "On the role of hand synergies in the optimal choice of grasping forces," Autonomous Robots, vol. 31, pp. 235-252, July 2011.

[34] D. Prattichizzo, M. Malvezzi, M. Gabiccini, and A. Bicchi, "On motion and force controllability of precision grasps with hands actuated by soft synergies," IEEE Trans. Robotics, vol. 29, no. 6, pp. 1440-1456, Dec. 2013.

[35] R. Suárez, J. Rosell, and N. García, "Using synergies in dual-arm manipulation tasks," in Proc. IEEE Int. Conf. Robotics and Automation, May 2015, pp. 5655-5661.

[36] N. García, R. Suárez, and J. Rosell, "Task-Dependent synergies for motion planning of an anthropomorphic dual-arm system," IEEE Trans. Robotics, vol. 33, no. 3, pp. 756-764, June 2017.

[37] J. Cui, Y. Liu, Y. Xu, H. Zhao, and H. Zha, "Tracking generic human motion via fusion of low- and high-dimensional approaches," IEEE
Trans. Systems, Man and Cybernetics: Systems, vol. 43, no. 4, pp. 9961002, July 2013.

[38] L. Colasanto, R. Suárez, and J. Rosell, "Hybrid mapping for the assistance of teleoperated grasping tasks," IEEE Trans. Systems, Man, and Cybernetics: Systems, vol. 43, no. 2, pp. 390-401, Mar. 2013.

[39] T. Kondo, O. Amagi, and T. Nozawa, "Proposal of anticipatory pattern recognition for EMG prosthetic hand control," in Proc. IEEE Int. Conf. Systems, Man and Cybernetics, Oct. 2008, pp. 897-902.

[40] N. García, J. Rosell, and R. Suárez, "Motion planning using first-order synergies," in Proc. IEEE/RSJ Int. Conf. Intelligent Robots and Systems, Sept. 2015, pp. 2058-2063.

[41] N. García, R. Suárez, and J. Rosell, "First-Order synergies for motion planning of anthropomorphic dual-arm robots," in Proc. IFAC World Congress, July 2017, pp. 2283-2290.

[42] M. Liarokapis, P. Artemiadis, and K. Kyriakopoulos, "Functional anthropomorphism for human to robot motion mapping," in Proc. IEEE Int. Symp. Robot and Human Interactive Communication, Sept. 2012, pp. 31-36.

[43] W. Chen, C. Xiong, and S. Yue, "Mechanical implementation of kinematic synergy for continual grasping generation of anthropomorphic hand," IEEE/ASME Trans. Mechatronics, vol. 20, no. 3, pp. 1249-1263, June 2015.

[44] T. Wimböck, B. Jan, and G. Hirzinger, "Synergy-level impedance control for a multifingered hand," in Proc. IEEE/RSJ Int. Conf. Intelligent Robots and Systems, Sept. 2011, pp. 973-979.

[45] J. Steffen, R. Haschke, and H. Ritter, "Towards dextrous manipulation using manipulation manifolds," in Proc. IEEE/RSJ Int. Conf. Intelligent Robots and Systems, Sept. 2008, pp. 2738-2743.

[46] B. Akgun and M. Stilman, "Sampling heuristics for optimal motion planning in high dimensions," in Proc. IEEE/RSJ Int. Conf. Intelligent Robots and Systems, Sept. 2011, pp. 2640-2645

[47] J. Rosell, A. Pérez, A. Aliakbar, Muhayyuddin, L. Palomo, and N. García, "The Kautham project: A teaching and research tool for robot motion planning," in Proc. IEEE Int. Conf. Emerging Technologies and Factory Automation, Sept. 2014.

[48] IOC-UPC. (2017, July) Human-Demonstrated Motion Planning for Anthropomorphic Dual-Arm Robots: Video of the experiments. [Online]. Available: https://sir.upc.edu/projects/human-demonstrated/index.html

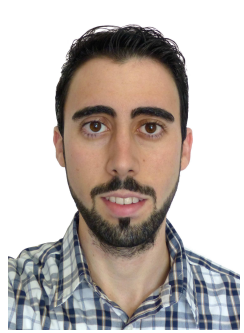

Néstor García received the B.S. degree (with honors) in Industrial Engineering from the "Universitat Politècnica de Catalunya" (UPC), Barcelona, Spain, in 2015. He is currently working toward the Ph.D degree in Automatic Control, Robotics and Computer Vision at the Institute of Industrial and Control Engineering (IOC), UPC. His current research interests are focused upon task and motion planning, human-robot interaction, multirobot cooperation and learning in robotic systems.

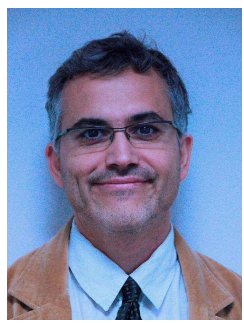

Jan Rosell received the BS degree in Telecommunication Engineering and the Ph.D. degree in Advanced Automation and Robotics from the "Universitat Politècnica de Catalunya" (UPC), Barcelona, Spain, in 1989 and 1998, respectively. He joined the Institute of Industrial and Control Engineering (IOC) in 1992 where he has developed research activities in robotics. He has been involved in teaching activities in Automatic Control and Robotics as Assistant Professor since 1996 and as Associate Professor since 2001. His current technical areas include task and motion planning, mobile manipulation, and robot co-workers

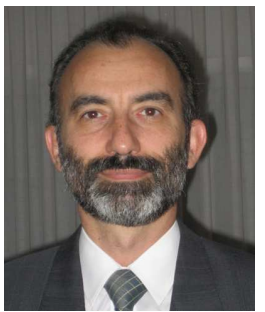

Raúl Suárez (M’96) received the Electronic Engineer degree (with honors) from the National University of San Juan, Argentina, in 1984, and the $\mathrm{Ph} . \mathrm{D}$. degree (cum laude) from the "Universitat Politècnica de Catalunya" (UPC), Barcelona, Spain, in 1993. He is Research Supervisor at the Institute of Industrial and Control Engineering (IOC), UPC, where he has been responsible for the research line on Process Control (1998-2003), Assistant Director (2003-2009) and Director (2009-2016) and the Coordinator of the Doctoral Program on Robotics (since 1995). His main research areas include grasping and manipulation, mechanical hands, fixturing, assembly, task planning, telemanipulation and manufacturing automation 